\title{
RECHERCHE DE LA D.L. 50 DE LA DELTAMÉTHRINE (DECIS) CHEZ MEGACHILE ROTUNDATA F. ABEILLE POLLINISATRICE DE LA LUZERNE (MEDICAGO SATIVA L.) ET DES EFFETS DE DOSES INFRALÉTHALES SUR LES ADULTES ET LES LARVES
}

\author{
J.N. TASEI, S. CARRE, B. MOSCATELLI, Catherine GRONDEAU \\ Laboratoire de Zoologie, I.N.R.A., 86600 Lusignan, France
}

RÉSUMÉ

La D.L. 50 de la deltaméthrine chez les deux sexes de l'abeille solitaire Megachile rotundata, a été évaluée en laboratoire chez les mâles et les femelles : $5 \times 10^{-3} \mu \mathrm{g} /$ abeille et $12 \times 10^{-3} \mu \mathrm{g} / \mathrm{abeille}$ respectivement. Les seuils d'innocuité (D.L. 1) sont respectivement : $1 \times 10^{-3} \mu \mathrm{g} / \mathrm{abeille}$ et $2 \times 10^{-3} \mu \mathrm{g} /$ abeille. Les doses inférieures à ces seuils réduisent la longévité des deux sexes. L'application de la D.L. 1 sur des femelles élevées en serre tend à réduire légèrement leur fécondité sans affecter leur longévité. L'ingestion par les larves de provisions contenant $1 \mathrm{ppm}$ et $0,1 \mathrm{ppm}$ de deltaméthrine, soit empêche le complet développement larvaire, soit le ralentit. La dose de $0,01 \mathrm{ppm}$ correspond à la concentration maximum relevée dans le pollen des nids bâtis après traitement de champ. Appliquée sur les provisions en laboratoire, elle ne cause aucun effet direct sur les larves.

\section{INTRODUCTION}

Des travaux sur l'effet des insecticides sur les apoïdes ont montré que certaines applications de produits à des doses inférieures au seuil de nocivité provoquaient des troubles variés qui perturbaient plus ou moins profondément la physiologie ou le comportement des individus. Chez les ouvrières d'Abeille domestique traitées au diazinon, Smirle et al. (1984) ont décelé une réduction de la longévité. En utilisant du parathion à faible dose, SHricker et STEPHEN (1970) ont noté une modification du rythme des « danses » qui permettent aux butineuses de transmettre à leurs congénères la localisation des sources de nourriture. La perméthrine est capable de réduire l'activité de butinage ainsi que les contacts antennaires des ouvrières selon Cox et WiLson (1984). Par contre, le fongicide dithane peut augmenter la récolte de pollen (BUCKNER et al., 1976). Le seul exemple publié de travail similaire sur abeille solitaire est celui de Torchio (1983) qui a démontré qu'un traitement au trichlorfon n'affectant pas la survie des mégachiles de la luzerne (Megachile rotundata F.) peut réduire considérablement la fécondité des femelles. 
Or, nos études récentes sur ce même apoïde solitaire ont laissé supposer que des traitements à base de deltaméthrine effectués dans les conditions naturelles pouvaient ne pas avoir d'effet léthal dans la journée suivant l'application du produit, mais étaient susceptibles de réduire la longévité et par conséquent la durée de ponte des femelles (TASEI et CARre, 1985).

L'étude présentée ici prend origine dans cette hypothèse ainsi que dans lobservation de Torchio (1983). Notre démarche a consisté à établir en premier lieu la «Dose léthale $50 »$ en laboratoire de la deltaméthrine pour les deux sexes de Megachile rotundata. Ces résultats nous procurant également la D.L. 1, il était ensuite possible de choisir une gamme de doses inférieures à ce seuil d'innocuité et de les appliquer à des mâles et des femelles afin de mesurer leur effet sur leur longévité et leur fécondité dans des conditions standardisées ou semi-naturelles.

Par ailleurs, divers auteurs ayant observé des perturbations chez les larves de $M$. rotundata, à la suite d'applications d'insecticides sur luzerne (WALler, 1969 ; George et Rincker, 1985 ; Tasei et Carre, 1985), il était nécessaire de connaître avec plus de précision l'effet sur les larves, de l'ingestion de quantités connues d'insecticide. Nous avons donc recherché le seuil d'innocuité de la deltaméthrine pour les larves de $M$. rotundata en laboratoire, puis nous avons mesuré l'effet de doses inférieures à ce seuil, sur leur développement.

\section{MATÉRIEL ET MÉTHODES}

Tous les insectes utilisés dans ces expériences ont pour origine un élevage de mégachiles canadiens du Saskatchewan. L'insecticide a été le DECIS à $25 \mathrm{~g} / 1$ de deltaméthrine.

1) Détermination de la D.L. 50 de la deltaméthrine pour les adultes de M. rotundata

Le principe que nous décrivons ici est emprunté à la méthode utilisée par Bos (1980) sur l'Abeille donıestique : dans une expérience préliminaire 5 doses d'une gamme de raison 10 , ont été appliquées à des groupes de 10 individus des deux sexes, âgés de moins de 48 heures. Ces doses étaient comprises entre $2 \times 10^{-4} \mu \mathrm{g}$ de deltaméthrine par abeille de $2 \mu \mathrm{g}$. Les mortalités observées au bout de 48 heures ont permis de situer approximativement la D.L. 50. L'expérience définitive a consisté à appliquer 7 doses comprises entre $5 \times 10^{-4} \mu \mathrm{g} /$ abeille et $3,2 \times 10^{-2} \mu \mathrm{g} /$ abeille pour les mâles et 7 doses comprises entre $2,5 \times 10^{-3} \mu \mathrm{g} / \mathrm{abeille}$ et $0,16 \mu \mathrm{g} /$ abeille pour les femelles. Chacune de ces gammes était de raison 2 . Chaque dose était appliquée à 90 mâles $(15 \times 6)$ et 75 femelles $(15 \times 5)$. Les insectes recevaient sur leur thorax une goutte d'1 $\mu \mathrm{l}$ de solution acétonique délivrée par une micropipette "Micropettor ", les témoins étant traités avec $1 \mu \mathrm{l}$ d'acétone pur. Après les applications, les abeilles étaient conservées dans des boîtes de plastique transparent à couvercle grillagé de $9 \times 6 \times 2 \mathrm{~cm}$, à $25^{\circ} \mathrm{C}$, durant 48 heures, jusqu'au moment du comptage des morts.

\section{2) Estimation de la longévité des adultes après l'application de doses infraléthales}

Les doses ont été choisies après la mesure de la D.L. 50 et de la D.L. 1. On a effectué des dilutions successives pour obtenir une gamme de concentrations comprises entre $8 \times 10^{-4} \mathrm{~g} / \mathrm{l}$ et $1,6 \times 10^{-6} \mathrm{~g} / \mathrm{l}$. 
Les applications ont été exécutées comme cela est décrit dans le $\$ 1$. Cependant, la longévité des mâles paraissant trop affectée par l'acétone, on a opté pour des applications d'une solution aqueuse contenant un mouillant (AS 2 de chez P.C.) à raison de $12 \mathrm{ml} / 1$. Les femelles ont reçu une solution acétonique. Les témoins, selon leur sexe, recevaient soit $1 \mu \mathrm{l}$ d'eau avec mouillant, soit $1 \mu \mathrm{l}$ d'acétone pur. Après traitement, les insectes ont été conservés dans les boîtes de plastique à couvercle grillagé mises en incubateur éclairé réglé à $25^{\circ} \mathrm{C}$. Chaque boîte contenait de 11 à 15 individus. Le nombre de répétitions a varié de 4 à 7 . Les insectes avaient la possibilité de s'alimenter dans des nourrisseurs contenant une solution sucrée à $30 \%$. Durant une dizaine de jours la mortalité à l'intérieur de chaque boîte a été évaluée journellement.

\section{3) Estimation de la fécondité après l'application d'une dose infraléthale de deltaméthrine}

Cette expérimentation a été réalisée dans un compartiment de serre vitrée dans lequel on a introduit une trentaine de femelles. La moitié était des femelles témoins sur le thorax desquelles avait été déposé $1 \mu \mathrm{l}$ d'eau contenant du mouillant AS $2(12 \mathrm{ml} / \mathrm{l})$. L'autre moitié des femelles recevait avant lintroduction en serre $1 \mu \mathrm{l}$ de solution aqueuse, avec mouillant, contenant $2 \times 10^{-3} \mu \mathrm{g}$ de deltaméthrine, soit une dose égale à la D.L. 1. Après application des solutions, les femelles étaient marquées avec un point de couleur sur le thorax afin que l'on distingue les traitées des témoins. Elles pouvaient s'accoupler avec des mâles, butiner sur une quarantaine de pieds de mélilot en fleurs et nidifier dans des tubes de bambou démontables disposés dans des nichoirs accrochés à $1,70 \mathrm{~m}$ du sol. Chaque jour avant l'envol des femelles hors de leur nid, les bambous étaient ouverts et l'on enregistrait le type de femelle qui y logeait, ainsi que l'état d'avancement du nid.

\section{4) Estimation des effets de doses de deltaméthrine appliquées sur la nourriture larvaire}

Afin d'obtenir une population homogène de larves de sexe mâle, nous avons procédé à un élevage de 120 femelles vierges dans deux enceintes de serre et dans un tunnel grillagé. Chaque jour les cellules approvisionnées et contenant un œuf étaient retirées des nichoirs et placées verticalement dans des boîtes individuclles en plastique transparent de $2 \times 2 \times 1 \mathrm{~cm}$. Lorsque les oufs ont été pondus en nombre suffisant ils ont été incubés à $30^{\circ} \mathrm{C}$ afin de faire éclore les larves. Dès leur éclosion les traitements ont été appliqués. Chacun d'entre eux a nécessité 60 jeunes larves $(4 \times 15)$. A la surface de chaque provision on a déposé $1 \mu \mathrm{l}$ de solution aqueuse d'insecticide, quatre doses ont été utilisées : $10^{-1} \mu \mathrm{g}$ de deltaméthrine/cellule, $10^{-2} \mu \mathrm{g}, 10^{-3} \mu \mathrm{g}$ et $10^{-4} \mu \mathrm{g}$. Etant donné que les provisions de pollen pèsent en moyenne $100 \mathrm{mg}$, les doses appliquées varient de $1 \mathrm{ppm}$ à $10^{-3} \mathrm{ppm}$. Après traitement, les larves ont poursuivi leur développement en incubateur réglé à $30^{\circ} \mathrm{C}$ et ont fait l'objet d'observations journalières sur la mortalité et le stade de développement (début et fin du filage du cocon puis nymphose éventuelle).

Des nids de mégachiles ont été prélevés dans les conditions naturelles dès leur construction avant et juste après le traitement du champ avec de la deltaméthrine à $7,5 \mathrm{~g} / \mathrm{ha}$. L'insecticide a été recherché dans le pollen. La limite de détection de l'analyse était de $10^{-3} \mathrm{ppm}$.

\section{RÉSULTATS}

\section{1) Calcul de la D.L. 50 et de la D.L. 1 chez les mâles et les femelles de mégachile}

Les tableaux 1 et 2 indiquent la précision des mesures de mortalité ainsi que les mortalités moyennes de chacun des traitements opérés avec les 7 concentrations choisies. Après une légère correction due aux mortalités enregistrées dans le témoin, nous avons appliqué la méthode recommandée par Busvine (1971) qui permet le calcul de la droite de régression des pourcentages de mortalité transformés en fonction des logarithmes des concentrations. 
TAB. 1. - Estimation de la dose léthale 50: Mortalité observée chez les mâles de mégachiles à $25^{\circ} \mathrm{C}, 48$ heures après application de 7 doses de deltaméthrine

TABL. 1. - Estimation of the L.D. 50: Mortality rate in male megachiles at $25^{\circ}$ C, 48 hours after application of 7 doses of deltamethrin

\begin{tabular}{|c|c|c|c|c|}
\hline $\begin{array}{c}\text { Dose } \\
\text { Dose rate }\end{array}$ & \multicolumn{4}{|c|}{$\begin{array}{c}\text { Mortalité } \\
\text { Mortality }\end{array}$} \\
\hline $\begin{array}{c}\text { ( } \mu \mathrm{g} \text { de matière } \\
\text { active par abeille) } \\
(\mu g \text { active } \\
\text { ingredient per bee) }\end{array}$ & $\begin{array}{c}\overline{\mathrm{X}} / \text { répétition } \\
\mathbf{X} / \text { replicate }\end{array}$ & $S^{2}$ & $\%$ & $\begin{array}{c}\% \text { corrigé selon } \\
\text { formule d'Abott } \\
\% \text { after Abott } \\
\text { correction }\end{array}$ \\
\hline $\begin{array}{l}0,032 \\
0,016 \\
0,008 \\
0,004 \\
0,002 \\
0,001 \\
0,0005\end{array}$ & $\begin{array}{r}14,7 \\
12,7 \\
8,2 \\
4,7 \\
3,2 \\
2,3 \\
1,0\end{array}$ & $\begin{array}{l}1,3 \\
3,2 \\
2,8 \\
3,2 \\
4,5 \\
1,9 \\
0,7\end{array}$ & $\begin{array}{r}97,7 \\
84,4 \\
54,4 \\
31,1 \\
21,1 \\
15,6 \\
6,7\end{array}$ & $\begin{array}{r}97,7 \\
84,3 \\
53,9 \\
30,3 \\
20,2 \\
14,6 \\
5,6\end{array}$ \\
\hline $\begin{array}{l}\text { Témoin (acétone) } \\
\text { Control (acetone) }\end{array}$ & 0,2 & & 1,1 & \\
\hline
\end{tabular}

TAB. 2. - Estimation de la dose léthale 50: Mortalité observée chez les femelles de mégachiles, à $25^{\circ} \mathrm{C}, 48$ heures après application de 7 doses de deltaméthrine

TABL. 2. - Estimation of the L.D. $50:$ Mortality rate in female megachiles, at $25^{\circ} \mathrm{C}$, 48 hours after application of 7 doses of deltamethrin

\begin{tabular}{|c|c|c|c|c|}
\hline \multirow{2}{*}{$\begin{array}{c}\text { Dose } \\
\text { Dose rate } \\
\begin{array}{c}(\mu \mathrm{g} \text { de matière } \\
\text { active par abeille } \\
(\mu \mathrm{g} \text { active } \\
\text { ingredient per bee) }\end{array}\end{array}$} & \multicolumn{4}{|c|}{$\begin{array}{l}\text { Mortalité } \\
\text { Mortality }\end{array}$} \\
\hline & $\begin{array}{c}\overline{\mathrm{X}} / \text { répétition } \\
\overline{\mathrm{X}} / \text { replicate }\end{array}$ & $S^{2}$ & $\%$ & $\begin{array}{c}\% \text { corrigé selon } \\
\text { formule d'Abott } \\
\% \text { after Abott } \\
\text { correction }\end{array}$ \\
\hline 0,16 & 15,0 & 0 & 100 & 100 \\
\hline 0,08 & 15,0 & 0 & 100 & 100 \\
\hline 0,04 & 14,4 & 0,2 & 96,0 & 95,9 \\
\hline 0,02 & 10,2 & 8,6 & 81,3 & 80,8 \\
\hline 0,01 & 3,8 & 10,2 & 25,3 & 23,3 \\
\hline 0,005 & 3,0 & 5,2 & 20,0 & 17,8 \\
\hline 0,0025 & 0,6 & 0,6 & 4,0 & 1,4 \\
\hline $\begin{array}{c}\text { Témoin (acétone) } \\
\text { Control (acetone) }\end{array}$ & 0,4 & & 2,7 & \\
\hline
\end{tabular}

Les calculs ont été facilités par l'utilisation d'un programme informatique. Les D.L. 50 sont respectivement pour les mâles et les femelles : $5,2 \times 10^{-3} \mu \mathrm{g}$ / abeille $\left(1,6 \times 10^{-3}<\right.$ D.L. $\left.50<17,5 \times 10^{-3}\right)$ et $12 \times 10^{-3} \mu \mathrm{g} /$ abeille $\left(3,7 \times 10^{-3}<\right.$ D.L. $\left.50<38,5 \times 10^{-3}\right)$. Les D.L. 1 sont respectivement pour les mâles et les femelles : $1 \times 10^{-3} \mu \mathrm{g} /$ abeille et $2 \times 10^{-3} \mu \mathrm{g} /$ abeille. 
2) Effets de doses infraléthales sur la longévité des adultes en conditions contrôlées

La figure 1 montre que les mâles ont commencé à mourir le $3^{\mathrm{e}}$ jour suivant le traitement. On note une accentuation de la mortalité le $5^{\mathrm{e}}$ jour et le $6^{\mathrm{e}}$ jour avec creusement des écarts entre les lots traités et le témoin. Les différences sont significatives $(P=0,05)$ le $5^{\mathrm{e}}$ jour pour les doses A $(0,8 \times$ D.L. 1$)$, B $(0,4 \times$ D.L. 1$)$, et C $(0,04 \times$ D.L. 1$)$. Le $6^{e}$ jour seule la dose $\mathrm{A}$ se distingue du témoin car ce dernier commence à subir les conséquences des conditions expérimentales ce qui est une incitation à l'arrêt de l'expérience (Tabl. 3). Si l'on se réfère à la figure 1, l'examen des courbes révèle que le taux de mortalité de $12 \%$ a été atteint par le témoin avec 3 jours de retard par rapport aux lots traités A, B, C. Autrement dit, ces doses ont réduit la longévité de 3 jours chez $12 \%$ des individus.

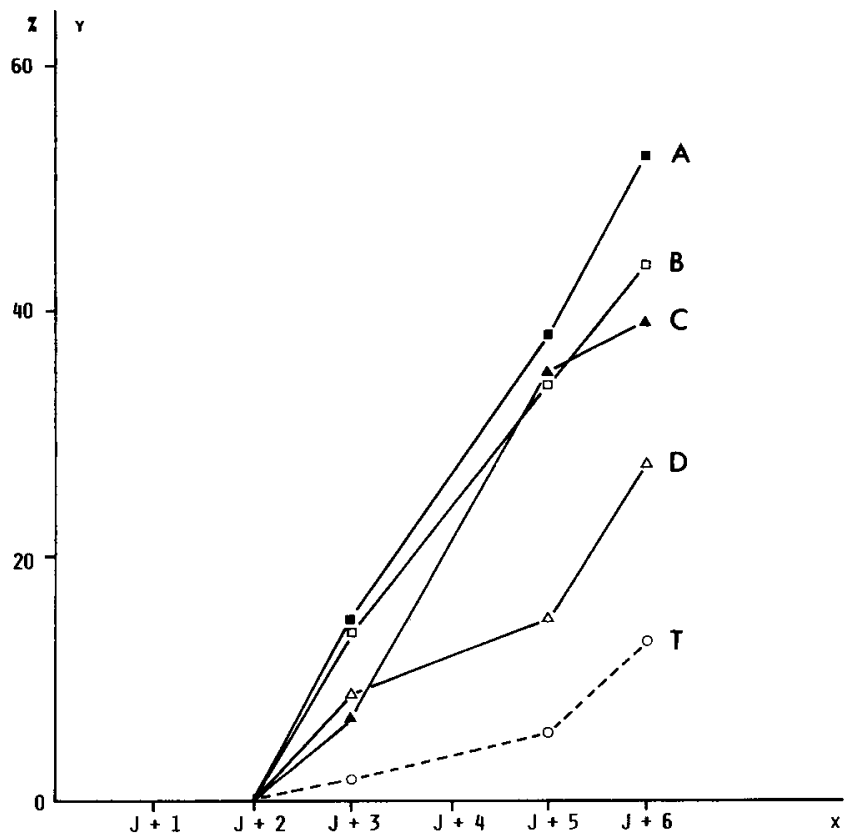

Fig 1. - Effet de l'application de doses infraléthales de deltaméthrine sur la mortalité des mâles de mégachiles en conditions contrôlées

FiG. 1. - Effect of sublethal doses of deltamethrin on the mortality of male megachiles in controlled conditions

\footnotetext{
$\mathbf{x}=$ Date

$y=\%$ morts

$\mathrm{A}=8 \times 10^{-4} \mu \mathrm{g} / \mathrm{abeille}$

$\mathrm{B}=4 \times 10^{-4} \mu \mathrm{g} /$ abeille

$\mathrm{C}=4 \times 10^{-5} \mu \mathrm{g} /$ abeille

$\mathrm{D}=4 \times 10^{-6} \mu \mathrm{g} /$ abeille

$\mathrm{T}=$ Témoin
}

$$
\begin{aligned}
& \mathrm{x}=\text { Date } \\
& \mathrm{y}=\% \text { dead } \\
& \mathrm{A}=8 \times 10^{-4} \mu \mathrm{g} / \mathrm{bec} \\
& \mathrm{B}=4 \times 10^{-4} \mu \mathrm{g} / \mathrm{bec} \\
& \mathrm{C}=4 \times 10^{-5} \mu \mathrm{g} / \text { bee } \\
& \mathrm{D}=4 \times 10^{-6} \mu \mathrm{g} / \mathrm{bee} \\
& \mathrm{T}=\text { Control }
\end{aligned}
$$


TAB. 3. - Effet de doses infraléthales de deltaméthrine sur les mâles de mégachiles à $25^{\circ} \mathrm{C}$, 5 à 6 jours après application

TABL. 3. - Effect of sublethal doses of deltamethrin on male megachiles at $25^{\circ} \mathrm{C}$, 5 and 6 days after treatment

\begin{tabular}{|c|c|c|c|c|c|c|c|}
\hline \multirow{3}{*}{$\begin{array}{c}\text { Traitement } \\
\text { Treatment }\end{array}$} & \multirow{3}{*}{$\begin{array}{c}\text { Dose ( } \mu \mathrm{g} \text { de } \\
\text { matière active } \\
\text { par abeille) } \\
\text { Dose rate ( } \mu \mathrm{g} \\
\text { of active } \\
\text { ingredient/bee) }\end{array}$} & \multicolumn{6}{|c|}{$\begin{array}{c}\text { Mortalité } \\
\text { Mortality }\end{array}$} \\
\hline & & \multicolumn{3}{|c|}{$J+5$} & \multicolumn{3}{|c|}{$J+6$} \\
\hline & & $\mathbf{N}$ & $\%$ & $\ll t »$ Dunnett ${ }^{1}$ & $\mathbf{N}$ & $\%$ & $\ll t »$ Dunnett ${ }^{2}$ \\
\hline A & $8 \times 10^{-4}$ & 40 & 38,1 & $3,09(\mathrm{~S})$ & 55 & 52,4 & $3,23(\mathrm{~S})$ \\
\hline B & $4 \times 10^{-4}$ & 36 & 34,3 & $2,81(\mathrm{~S})$ & 46 & 43,8 & 2,52 \\
\hline $\mathrm{C}$ & $4 \times 10^{-5}$ & 37 & 35,2 & $2,72(\mathrm{~S})$ & 41 & 39,0 & 2,13 \\
\hline D & $4 \times 10^{-6}$ & 16 & 15,2 & 0,91 & 35 & 33,3 & 1,65 \\
\hline \multicolumn{2}{|l|}{$\begin{array}{l}\text { Témoin (eau } \\
+ \text { mouillant) } \\
\text { Control (water } \\
+ \text { Wetting } \\
\text { Agent) }\end{array}$} & 6 & 5,7 & & 14 & 13,3 & \\
\hline \multicolumn{8}{|c|}{$\begin{array}{l}1 \mathrm{~F}=3,76(\mathrm{P}=0,016) . \\
2 \mathrm{~F}=2,94(\mathrm{P}=0,041) . \\
\text { valeur limite } \mathrm{t}=2,61 \text { pour } \mathrm{P}=0,05 \\
\text { " } \mathrm{t} \text { » limit value }=2,61 \text { at } \mathrm{P}=0,05\end{array}$} \\
\hline
\end{tabular}

Chez les femelles l'incidence des traitements sur les insectes n'est sensible qu'à partir du $7^{\mathrm{e}}$ jour (Fig. 2). Les $9^{\mathrm{e}}$ et $10^{\mathrm{e}}$ jours les écarts de mortalité entre la dose $\mathrm{A}(0,4 \times$ D.L. 1$)$ et le témoin sont les seuls qui sont significatifs (Tabl. 4). Le $12^{\mathrm{e}}$ jour, le témoin commençant à souffrir des conditions de claustration, il ne peut plus servir de référence. Les taux de mortalité compris entre $8 \%$ et $18 \%$ ont été atteints avec la dose $\mathrm{A}$ avec 3 à 4 jours d'avance sur le témoin. La réduction de longévité est donc du même ordre que chez les mâles.

3) Effet d'une dose infraléthale sur la fécondité des femelles élevées en serre

Les observations effectuées sur les 15 femelles traitées et les 15 femelles témoins ont permis de calculer le nombre de cellules d'élevage construites et le nombre d'œufs pondus par chaque type de femelle durant des périodes de temps de 4 à 6 jours (ligne I du tableau 5). A chaque période le nombre de femelles survivantes a été noté (ligne II). Les données précédentes des lignes I et II, sont les éléments nécessaires au calcul du nombre moyen de cellules à œuf construites par femelle (ligne III). Globalement en 5 semaines les 15 


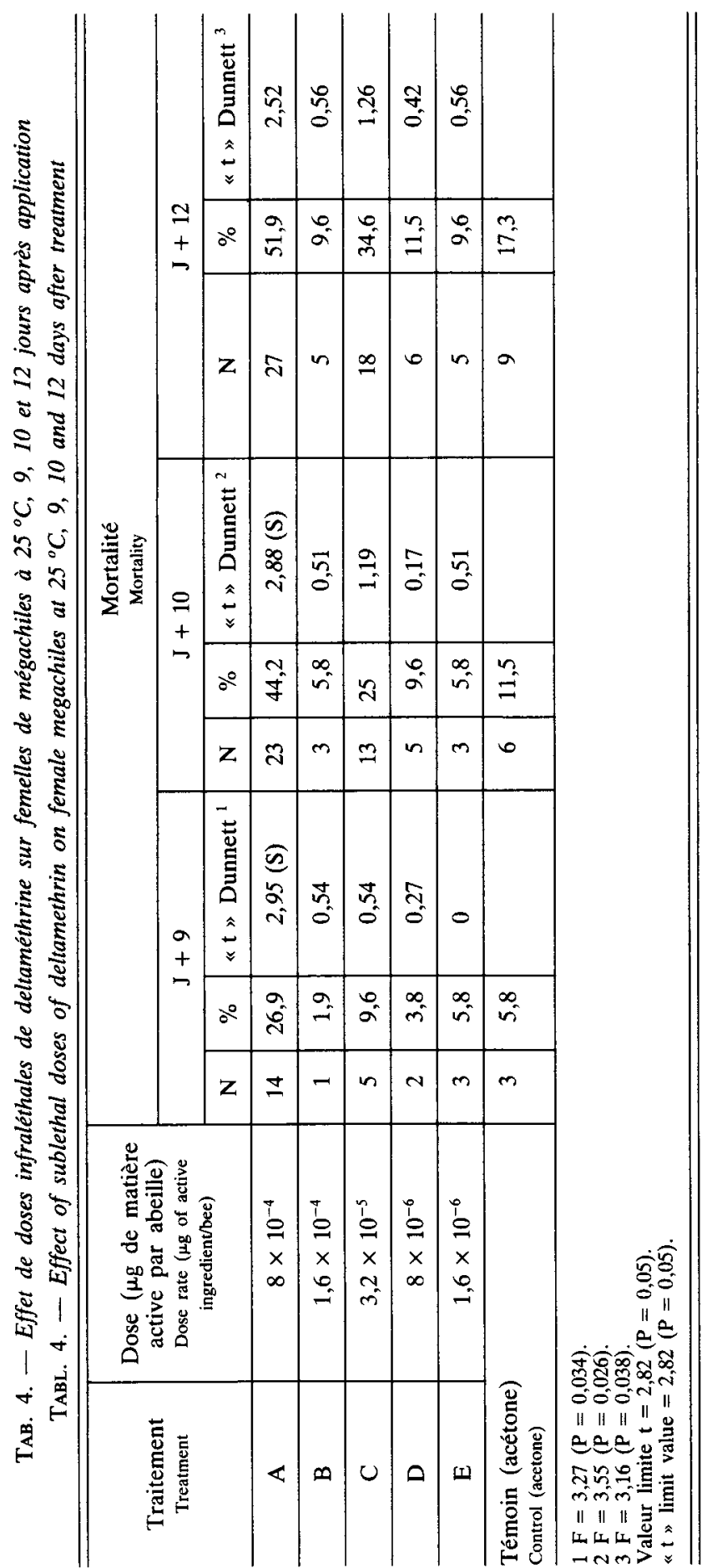




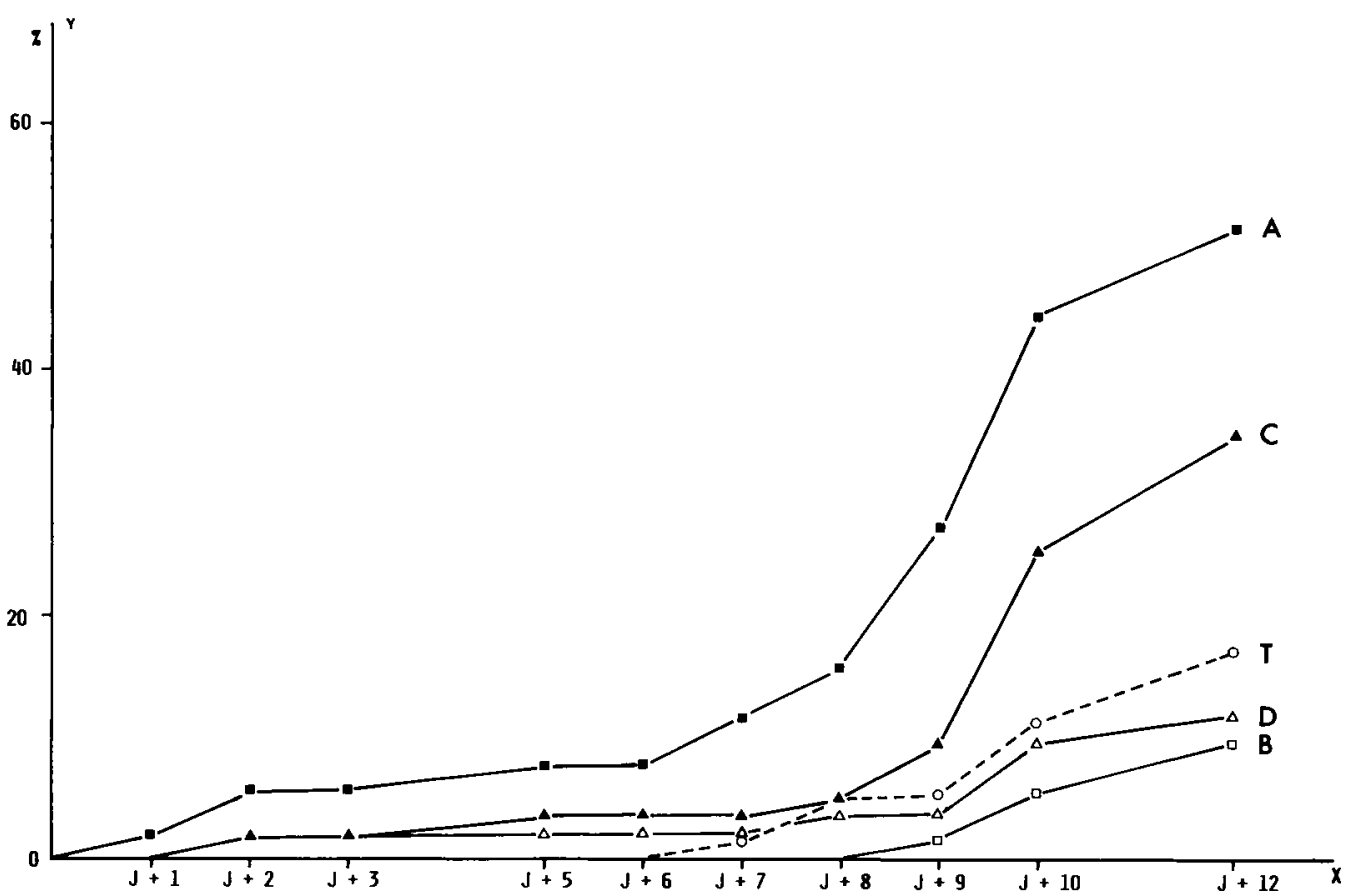

FIG 2. - Effet de l'application de doses infraléthales de deltaméthrine sur la mortalité des femelles de mégachiles en conditions contrôlées

FIG. 2. - Effect of sublethal doses of deltamethrin on the mortality of female megachiles in controlled conditions

$\mathbf{x}=$ Date

$\mathrm{y}=\%$ dead

$A=8 \times 10^{-4} \mu \mathrm{g} /$ bee

$\mathrm{B}=1.6 \times 10^{-4} \mu \mathrm{g} / \mathrm{bee}$

$\mathrm{C}=3.2 \times 10^{-5} \mu \mathrm{g} /$ bee

$\mathrm{D}=8 \times 10^{-6} \mu \mathrm{g} / \mathrm{bee}$

$\mathbf{T}=$ Control

$$
\begin{aligned}
& \mathrm{x}=\text { Date } \\
& \mathrm{y}=\% \text { morts } \\
& \mathrm{A}=8 \times 10^{-4} \mu \mathrm{g} / \text { abeille } \\
& \mathrm{B}=1,6 \times 10^{-4} \mu \mathrm{g} / \text { abeille } \\
& \mathrm{C}=3,2 \times 10^{-5} \mu \mathrm{g} / \text { abeille } \\
& \mathrm{D}=8 \times 10^{-6} \mu \mathrm{g} / \text { abeille } \\
& \mathrm{T}=\text { Témoin }
\end{aligned}
$$

femelles témoins ont construit 240 cellules, soit 16 par femelle, et les 15 femelles traitées 200 , soit 13 par femelle. L'analyse des données, durant les 7 périodes consécutives, par la méthode $\mathrm{du}$ " $\mathrm{t}$ apparié " montre que la fécondité des femelles témoins avait tendance à être plus forte que celle des femelles traitées. En effet, le «t» calculé $=2,21$ est compris entre les valeurs des seuils à 0,05 et 0,10 de probabilité.

4) Effet de l'ingestion par les larves, de faibles doses de deltaméthrine

A $30{ }^{\circ} \mathrm{C}$ les larves qui accomplissaient l'intégralité de leur développement ingéraient leurs provisions en 4 à 6 jours, puis filaient leur cocon en 3 à 
TAB. 5. - Effet de l'application d'une dose infraléthale de $2 \times 10^{-3} \mu \mathrm{g} /$ abeille de deltaméthrine sur la ponte des femelles de mégachiles élevées en serre

TABL. 5. - Effects of a sublethal dose application $\left(2 \times 10^{-3} \mu \mathrm{g} /\right.$ bee $)$ of deltamethrin on the fecundity of female megachiles reared in glasshouse

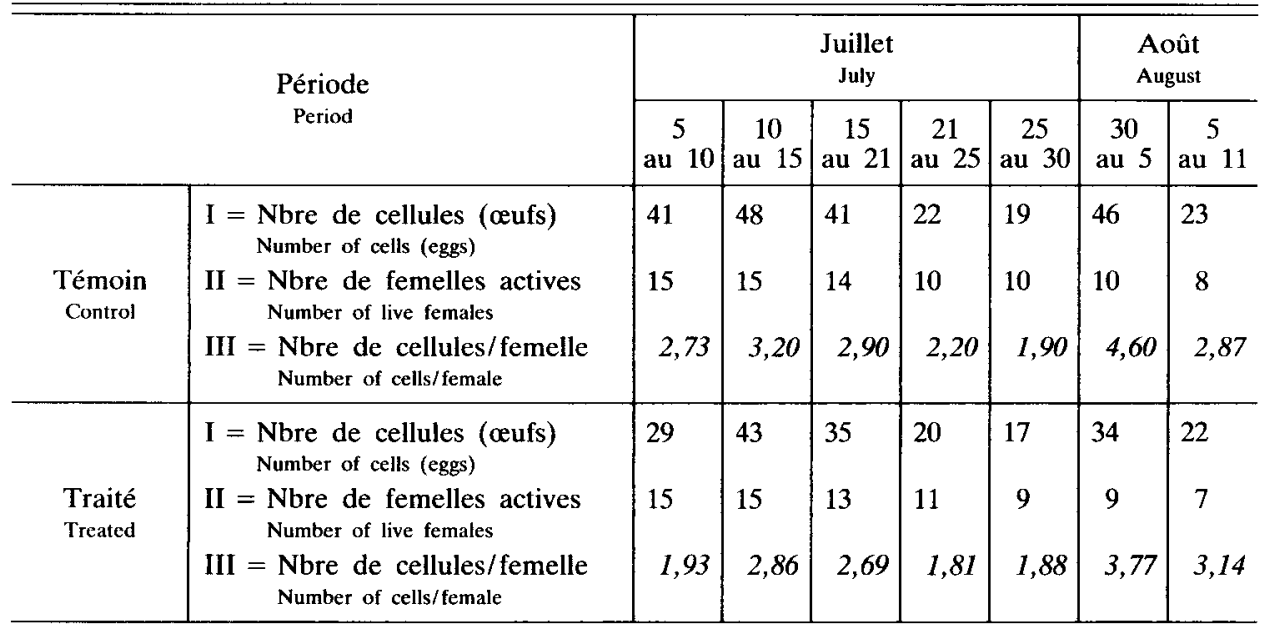

« $t$ " apparié « III témoin / III traité » $=2,21(\mathrm{P}<10 \%)$.

Paired « $t$ " test «III control / III treated " $=2,21(\mathrm{P}<0,1)$.

Тав. 6. - Effet de 4 doses de deltaméthrine, appliquées sur les provisions polliniques, sur la mortalité larvaire estimée à la fin du filage du cocon

TABL. 6. - Effect of 4 doses of deltamethrin applied to pollen provisions, on larval mortality estimated at the end of cocoon spinning

\begin{tabular}{|c|c|c|c|c|c|}
\hline \multirow{2}{*}{$\begin{array}{c}\text { Traitement } \\
\text { Treatment }\end{array}$} & \multirow{2}{*}{$\begin{array}{l}\text { Dose (mg de matière } \\
\text { active par kg } \\
\text { de provision }{ }^{\mathrm{T}} \text { ) } \\
\text { Dose rate (mg of active } \\
\text { ingredient per } \mathrm{kg} \\
\text { of provision }{ }^{\mathrm{t}} \text { ) }\end{array}$} & \multicolumn{2}{|c|}{$\begin{array}{c}\text { Mortalité } \\
\text { Mortality }\end{array}$} & \multirow{2}{*}{$\begin{array}{l}\text { Test du « F" } \\
\text { de Fisher } \\
\text { Fisher } * F * \text { test }\end{array}$} & \multirow{2}{*}{$\ll t »$ Dunnett ${ }^{2}$} \\
\hline & & $\mathbf{N}$ & $\%$ & & \\
\hline A & 1 & 33 & 55,0 & \multirow{4}{*}{$\begin{array}{l}F=6,45 \\
P=0,006\end{array}$} & $\mathrm{t}=4,82 ; \mathrm{S}$ \\
\hline B & $10^{-1}$ & 19 & 33,3 & & $\mathrm{t}=2,48 ; \mathrm{NS}$ \\
\hline $\mathrm{C}$ & $10^{-2}$ & 13 & 23,2 & & $\mathrm{t}=1,45 ; \mathrm{NS}$ \\
\hline D & $10^{-3}$ & 14 & 23,7 & & $\mathrm{t}=1,35 ; \mathrm{NS}$ \\
\hline $\begin{array}{l}\text { Témoin } \\
\text { Control }\end{array}$ & & 8 & 14,5 & & \\
\hline \multicolumn{6}{|c|}{$\begin{array}{l}1 \text { Poids moyen d'une provision pollinique }=100 \mathrm{mg} \text {. } \\
2 \text { Valeur limite } t=2,81 \text { pour } P=0,05 \\
1 \text { Mean weight of a pollen provision }=100 \mathrm{mg} \text {. } \\
2 \text { « } \gg \text { limit value }=2,81 \text { at } P=0,05\end{array}$} \\
\hline
\end{tabular}


6 jours. La durée de vie de celles qui mouraient n'était jamais inférieure à 4 jours. Plus de la moitié de la mortalité larvaire a été observée avant le début du filage du cocon, c'est-à-dire dans les 4 à 6 jours qui ont suivi l'éclosion de la larve et après ingestion d'une partie seulement des provisions polliniques. Les autres cas de mortalités sont survenus au cours du filage du cocon et très rarement après la fin.

TAB. 7. - Effet de 4 doses de deltaméthrine, appliquées sur les provisions polliniques, sur la durée du développement larvaire de l'éclosion à la fin du filage du cocon

TABL. 7. - Effect of 4 doses of deltamethrin applied to pollen provisions, on the length of the larval development from hatching to the end of cocoon spinning

\begin{tabular}{|c|c|c|c|c|}
\hline $\begin{array}{c}\text { Traitement } \\
\text { Treatment }\end{array}$ & $\begin{array}{c}\text { Dose (mg de matière } \\
\text { active par } \mathrm{kg} \\
\text { de provision) } \\
\text { Dose rate (mg of active } \\
\text { ingredient per kg } \\
\text { of provision) }\end{array}$ & $\mathrm{N}$ & $\begin{array}{c}\text { Durée du } \\
\text { développement larvaire } \\
\text { (jours) à } 30^{\circ} \mathrm{C} \\
\text { Length of larval } \\
\text { development (days) } \\
\text { at } 30^{\circ} \mathrm{C}\end{array}$ & $\begin{array}{c}\ll t » \text { de Student } \\
\text { Traitement/Témoin } \\
\text { Student } \& t » \\
\text { test treatment/control }\end{array}$ \\
\hline A & 1 & 28 & 10,5 & $\begin{array}{c}\mathbf{t}=3,59 \\
\mathbf{P}<0,001\end{array}$ \\
\hline B & $10^{-1}$ & 38 & 9,7 & $\begin{array}{r}\mathrm{t}=2,37 \\
\mathrm{P}<0,05\end{array}$ \\
\hline$C$ & $10^{-2}$ & 43 & 8,9 & NS \\
\hline D & $10^{-3}$ & 45 & 8,5 & NS \\
\hline $\begin{array}{l}\text { Témoin } \\
\text { Control }\end{array}$ & & 47 & 8,6 & \\
\hline
\end{tabular}

TAB. 8. - Effet de 4 doses de deltaméthrine, appliquées sur les provisions polliniques, sur le taux de diapause larvaire

TABL. 8. - Effect of 4 doses of deltamethrin applied to the pollen provisions, on the rate of larval diapause

\begin{tabular}{|c|c|c|c|c|c|}
\hline $\begin{array}{l}\text { Traitement } \\
\text { Treatment }\end{array}$ & $\begin{array}{c}\text { Dose (mg de } \\
\text { matière active } \\
\text { par kg } \\
\text { de provision) } \\
\text { Dose rate } \\
\text { (mg of active } \\
\text { ingredient } \\
\text { per kg } \\
\text { of provision) }\end{array}$ & $\begin{array}{c}\text { Effectif } \\
\text { des larves } \\
\text { en diapause } \\
\text { Number of } \\
\text { diapausing } \\
\text { larvae }\end{array}$ & $\begin{array}{c}\text { Effectif } \\
\text { des larves } \\
\text { survivantes } \\
\text { Number } \\
\text { of surviving } \\
\text { larvae }\end{array}$ & $\begin{array}{c}\% \text { de diapause } \\
\text { Diapause rate }\end{array}$ & $\begin{array}{c}\text { Test «F» } \\
\text { de Fisher } \\
\text { Fisher « } F » \\
\text { test }\end{array}$ \\
\hline A & 1 & 22 & 27 & 81,5 & \\
\hline B & $10^{-1}$ & 24 & 38 & 63,1 & $F=2,11$ \\
\hline $\mathrm{C}$ & $10^{-2}$ & 34 & 43 & 79,1 & $\mathbf{P}=0,14$ \\
\hline D & $10^{-3}$ & 27 & 45 & 60,0 & \\
\hline $\begin{array}{l}\text { Témoin } \\
\text { Control }\end{array}$ & & 28 & 47 & 59,6 & \\
\hline
\end{tabular}


Dans les tableaux 6 et 7 , il apparaît que la dose A soit $1 \mathrm{mg}$ de deltaméthrine par $\mathrm{kg}$ de provision pollinique a eu des effets évidents sur la survie des larves puisque $55 \%$ d'entre elles contre $14 \%$ chez le témoin ne sont pas arrivées à la fin du filage du cocon. Les larves survivantes ont eu une durée de développement supérieure de 2 jours à celle du témoin.

La dose $\mathrm{B}$, soit $0,1 \mathrm{mg}$ de deltaméthrine par $\mathrm{kg}$ de provision pollinique a tendu aussi à réduire la survie larvaire, bien que de façon moins prononcée que la dose $\mathrm{A}(\ll \mathrm{t} »$ non significatif pour $\mathrm{P}=0,05)$. En effet, la mortalité des larves dans ce $2^{\mathrm{e}}$ traitement a été de $33 \%$. De plus, la dose $\mathrm{B}$ a allongé la durée du développement d'un jour par rapport au témoin.

Les doses inférieures $C=10^{-2} \mathrm{mg} / \mathrm{kg}$ et $\mathrm{D}=10^{-3} \mathrm{mg} / \mathrm{kg}$ n'ont eu aucun effet significatif sur le développement des larves.

L'analyse de résidus de deltaméthrine des provisions de pollen de mégachiles contaminées dans les conditions naturelles a permis de déceler que la nourriture larvaire récoltée le lendemain du traitement contenait $10^{-2} \mathrm{mg}$ d'insecticide par $\mathrm{kg}$ de pollen (dose expérimentale $\mathrm{C}$ ). Les prélèvements correspondant à une récolte du pollen 5 à 6 jours après le lendemain du traitement contenaient la même quantité de deltaméthrine.

Les larves survivantes de tous les lots, soit se sont nymphosées, soit sont entrées en diapause. Le tableau 8 montre qu'avec nos effectifs sans doute trop faibles les différences de taux de diapause entre traitements et témoin ne se sont pas révélées significatives. Seules les doses $A(1 \mathrm{mg} / \mathrm{kg})$ et $C\left(10^{-2} \mathrm{mg} /\right.$ $\mathrm{kg}$ ) pourraient avoir un effet positif sur l'induction de la diapause.

\section{DISCUSSION - CONCLUSION}

1) Si l'on tient compte du poids moyen des mâles de mégachiles : $25 \mathrm{mg}$ et des femelles : $32 \mathrm{mg}$, la D.L. 50 est de $0,2 \mu \mathrm{g} / \mathrm{g}$ chez les mâles et $0,4 \mu \mathrm{g} / \mathrm{g}$ chez les femelles à $25^{\circ} \mathrm{C}$. Ces doses sont à comparer avec la D.L. 50 de l'abeille domestique, qui selon Bós et MAsson (1982) est comprise à $32^{\circ} \mathrm{C}$ entre 0,1 et $0,3 \mu \mathrm{g} / \mathrm{g}$ et qui est réduite de moitié à $20^{\circ} \mathrm{C}$. Etant donné la variation des résultats en fonction de la température et la précision de la méthode, il n'est pas possible de conclure sur la sensibilité intrinsèque relative de l'abeille domestique et des mégachiles.

2) Nos essais montrent que des doses de deltaméthrine inférieures au seuil théorique d'innocuité et appliquées sur le thorax d'adultes maintenus en confinement étroit, peuvent perturber leur longévité. Les mâles manifestent une plus grande sensibilité que les femelles. En effet, leur longévité est 
affectée par une dose 40 fois inférieure à la D.L. 1 et 100 fois inférieure à la D.L. 50, alors que les femelles ne réagissent qu'à la dose moitié de la D.L. 1 .

Il est probable que les conditions de claustration exacerbent ces phénomènes puisque l'application de la D.L. 1 sur des femelles en serre ne modifie pas leur longévité.

3) La diminution de l'intensité de ponte qui semble consécutive à l'application de $0,002 \mu \mathrm{g} /$ abeille reste constante durant la plus grande partie de la vie des femelles. La réduction de la fécondité moyenne de l'ordre de $20 \%$ est nettement inférieure à celle de $50 \%$ qui était observée par ToRCHIO (1983) après un traitement au trichlorfon à faible dose. Il est cependant à noter que l'auteur n'a pas poursuivi ses observations après le $4^{\mathrm{e}}$ jour suivant l'application.

4) Le développement ne peut s'achever pour $40 \%$ des larves lorsqu'elles s'alimentent sur des provisions contenant $1 \mathrm{ppm}$ de deltaméthrine. Si la concentration d'insecticide n'est que de $0,1 \mathrm{ppm}$, il est encore possible de détecter le même effet sur près de $20 \%$ des larves. Dans les 2 cas, les larves qui achèvent leur développement le font à un rythme significativement plus lent que les larves témoins. Les concentrations de $0,01 \mathrm{ppm}$ et $0,001 \mathrm{ppm}$ ne provoquent d'effet ni sur la survie des larves ni sur leur vitesse de développement. Or les contaminations naturelles du pollen avec la deltaméthrine ne semblent pas dépasser le seuil de $0,01 \mathrm{ppm}$ dans les nids de mégachiles et un essai décrit par TASEr et al. (1987 b) démontre que les larves nourries sur ces provisions ont un taux de survie identique à celui des témoins. Chez les larves, le seuil d'innocuité par ingestion est donc compris entre $0,01 \mathrm{ppm}$ et $0,1 \mathrm{ppm}$ de deltaméthrine.

5) L'espèce $M$. rotundata a confirmé qu'elle était un bon modèle d'apoïde solitaire, permettant de répéter aisément des expériences, de développer une méthodologie et d'acquérir de nouvelles données écotoxicologiques chez les insectes pollinisateurs.

6) Nos résultats, ainsi que les travaux déjà cités, sont autant d'incitations à ne pas seulement considérer les mortalités immédiates dues à l'intoxication aiguë des insecticides. Il faut aussi, lorsque l'on juge comparativement les effets des traitements phytosanitaires sur la faune pollinisatrice, prendre en compte les conséquences multiples, à manifestation discrète et apparaissant à moyen terme, de l'application de doses d'insecticides inférieures au seuil d'innocuité. L'étude en laboratoire est souvent indispensable à la mise en évidence des phénomènes, mais la confirmation des résultats dans les conditions naturelles est nécessaire à une juste évaluation des risques encourus par les insectes pollinisateurs à la suite d'applications d'insecticides sur les cultures en fleurs. 


\section{REMERCIEMENTS}

Nous sommes redevables à M. J.C. Moreteau (Université de Paris-Sud) ainsi que $M^{\text {lle }}$ Isabelle Badenhausser (I.N.R.A., Lusignan) pour la cession du programme de calcul informatique de la Dose léthale 50, ainsi que sa mise en cuvre.

Nous remercions la Société ROUSSEL-UCLAF qui a financé les analyses de résidus de deltaméthrine.

\section{SUMMARY}

\section{RESEARCH OF THE L.D. 50 OF DELTAMETHRIN ON THE ALFALFA LEAFCUTTING BEE : MEGACHILE ROTUNDATA AND THE EFFECTS OF SUBLETHAL DOSES ON ADULTS AND LARVAE}

We studied the effects of sublethal doses of deltamethrin on $M$. rotundata by greenhouse and laboratory tests.

\section{Material and methods}

1) The L.D. 50 and L.D. 1 were calculated from the regression line fitted to dosage mortality data on males and females samples established at $25^{\circ} \mathrm{C}$. Dead individuals were counted 2 days after the topical application of doses.

2) Four doses less than the L.D. 1 were applied to males and females $(1 \mu \mathrm{l}$ of solution on thorax) which were kept in controlled conditions $\left(25^{\circ} \mathrm{C}\right.$ and constant light) and fed with sugar syrup. The effects of doses on the life length of insects were estimated by daily observations of mortality.

3) In a glasshouse with plants of blooming sweetclover we reared 15 control females and 15 females treated with a topical application equal to the L.D. 1. The mean number of eggs laid by each group of females were estimated by daily observations of nest building and counts of surviving bees.

4) The effects of 4 doses of deltamethrin deposited in pollen provisions were studied on male immature stages in controlled conditions $\left(30^{\circ} \mathrm{C}\right)$.

Results

1) The L.D. 1 is $1 \times 10^{-3} \mu \mathrm{g}$ of deltamethrin per male bee and $2 \times 10^{-3} \mu \mathrm{g}$ per female bee. The L.D. 50 is respectively for both sexes : $5 \times 10^{-3} \mu \mathrm{g} /$ bee and $12 \times 10^{-3} \mu \mathrm{g} /$ bee (Table 1,2).

2) In controlled conditions, applications of doses inferior to the L.D. 1 to males and females resulted in the reduction of life length of bees. Males were more susceptible than females to doses lower than this threshold. The shortening of life length that was noted in controlled conditions could not be observed in the glasshouse (Table 3, 4 and Fig. 1,2).

3) When $2 \times 10^{-3} \mu \mathrm{g}$ of deltamethrin per bee was applied to females they laid $20 \%$ less eggs than control females. This effect was nearly constant during the 6 week flight (Table 5).

4) When fed on pollen provisions contaminated with $1 \mathrm{mg} / \mathrm{kg}$ of deltamethrin $40 \%$ of the larvae could not reach the final stage. With $0.1 \mathrm{mg} / \mathrm{kg}$ the same effects were observed at a lower rate. In both cases, surviving larvae which completed their cocoon developed less rapidly than the control. The L.D. 1 lies betwcen $10^{-1} \mathrm{mg} / \mathrm{kg}$ and $10^{-2} \mathrm{mg} / \mathrm{kg}$ (Table 6,7 ). After natural contamination with deltamethrin maximum concentration of residues was $10^{-2} \mathrm{mg} / \mathrm{kg}$.

It is important to consider both the short term acute toxicity of insecticides on adult bees and the various long term effects due to metabolic changes induced by doses inferior to the L.D. 1 threshold. 


\section{ZUSAMMENFASSUNG \\ BESTIMMUNG DER L.D. 50 VON DELTAMETHRIN (DECIS) \\ BEI MEGACHILE ROTUNDATA F, DER FÜR DIE BESTÄUBUNG DER LUZERNE (MEDICAGO SATIVA) SPEZIALISIERTEN BIENE, UND DIE WIRKUNG INFRALETALER DOSEN AUF ADULTE UND LARVEN}

Auf der Grundlage der Arbeiten verschiedener Autoren über die Wirkung von subletalen Dosen von Insektiziden auf Bienen haben wir in Glashaus- und Laborversuchen die Folgen von unterhalb der Schadschwelle liegenden Deltamethrin-Dosen auf Megachile rotundata untersucht.

\section{Material und Methoden}

- Die Bestimmung der L.D. 50 und der L.D. 1 erfolgte bei $25^{\circ} \mathrm{C}$ durch Noticrung der Mortalität nach $48 \mathrm{~h}$ bei Gruppen von Männchen und Weibchen, die 7 verschiedene Dosen der Substanz erhalten hatten. Die Berechnung von Regressionsgeraden ermöglichte die Bestimmung der verschiedenen Schwellen.

- Vier Dosen unterhalb der L.D. 1 wurden auf Männchen und Weibchen angewandt $(1 \mu \mathrm{lder}$ Lösung auf den Thorax), die in einem beleuchteten Brutschrank bei $25^{\circ} \mathrm{C}$ gehalten wurden. Dic Ernährung der Tiere erfolgte mit Zuckerwasser. Tägliche Beobachtung der Mortalität erlaubte die Feststellung der Wirkung dieser Dosen auf die Lebensdauer der Adulten.

- In einem Glashaus mit blühendem Melilotus wurden 15 Weibchen gehalten, auf deren Thorax eine Dosis von $2 \times 10^{-3}$ aufgebracht worden war, und außerdem 15 unbehandelte Weibchen als Kontrolle. Die tägliche Kontrolle der Konstruktion von Nestern und der Zahl der Überlebenden lieferte die Elemente zur Berechnung der mittleren Intensität der Eiablage der beiden Gruppen von Bienen.

- Die Wirkung der Aufnahme von künstlich mit vier Dosen Deltamethrin kontaminierten Pollen auf Larven wurde im Laboratorium bei $30^{\circ} \mathrm{C}$ untersucht. Um die Untersuchung auf ein Geschlecht (Männchen) zu beschränken, wurden die Larven einer Zucht virgineller Weibchen entnommen.

\section{Resultate}

1) Die L.D. 1 beträgt $1 \times 10^{-3} \mu \mathrm{g} /$ Biene für Männchen und $2 \times 10^{-3} \mu \mathrm{g} /$ Biene für Weibchen ; die entsprechenden Dosen für die L.D. 50 sind $5 \times 10^{-3} \mu \mathrm{g} /$ Biene und $12 \times 10^{-3} \mu \mathrm{g} / \mathrm{Biene}$ (Tab. 1 und 2).

2) Im Laboratorium zeigten die Männchen und Weibchen, die Dosen unterhalb der Schadschwelle erhalten hatten, gegenüber den Kontrollen eine verkürzte Lebensdauer. Die Männchen zeigten sich für diese unterschwelligen Dosen empfindlicher als die Weibchen. Die verkürzende Wirkung auf die Lebensdauer der Weibchen, die sich unter den standardisierten Bedingungen des Laboratoriums deutlich zeigte, konnte unter den Bedingungen des Glashauses nicht nachgewiesen werden (Tab. 3 und 4 ; Fig. 1 und 2).

3) Die Fruchtbarkeit der Weibchen ist im Mittel um $20 \%$ verringert, wenn eine Dosis von $2 \times 10^{-3} \cdot \mu \mathrm{g} /$ Biene angewandt wurde. Die Verringerung der Intensität der Eiablage ist für die ganze Lebensdauer ungefähr konstant, selbst wenn diese 6 Wochen beträgt (Tab. 5).

4) Wenn sich die Larven mit Pollen ernähren, der $1 \mathrm{mg} / 1 \mathrm{~kg}$ Deltamethrin enthält, können $40 \%$ von ihnen ihre Entwicklung nicht vollenden. Dieselben Störungen, nur geringeren Ausmaßes, werden beobachtet, wenn die Konzentration $10 \mathrm{mal}$ geringer ist. In beiden Fällen erfolgt die Entwicklung der Larven, die sie bis zum Abschluß durchlaufen, langsamer als bei den Kontrollen. Die Schadschwelle verläuft zwischen $10^{-1} \mathrm{mg} / \mathrm{kg}$ und $10^{-2} \mathrm{mg} / \mathrm{kg}$ (Tab. 6 und 7). Bei natürlicher Kontamination mit Deltamethrin erreicht die maximale Konzentration des Rückstandes $10^{-2} \mathrm{mg} / \mathrm{kg}$.

5) Man muß nicht nur die unmittelbare Mortalität bei den adulten Bienen berücksichtigen, also die akute Toxizität des Insektizids, sondern auch die vielfältigen und versteckten Erscheinungen während eines mittleren Zeitabschnittes, verursacht durch Störungen des Stoffwechsels auf Grund von Dosen unterhalb der Schadschwelle (L.D. 1). 


\section{RÉFÉRENCES BIBLIOGRAPHIQUES}

Bos Ch., 1980. - Mise au point des données récentes sur le mode d'action des insecticides (pyréthrinoïdes) en particulier sur les abeilles (Rapport d'A.E.A., Univ. Paris VII).

Bos Ch., Masson C., 1982. - Toxicity and repellent effect of the synthetic pyrethroids on bees: methodological aspects. Report of the meeting. $2^{\text {nd }}$ Symp. on the harmonization of methods for testing the toxicity of pesticides to bees, Hohenheim, R.F.A., 21-23 Sept. 1982, Ap.9.

Buckner C.H., McLeod B.B., Gochnauer T.A., Lough R., 1976. - Effects of the fungicide, Dithane M-45 on colonies of honey bees, Apis mellifera L. Manitoba Entomol., 10, 46-48.

Busvine J.R., 1971. - A critical review of the techniques for testing insecticides. C.A.B. London.

Cox R.L., WiLson W.T., 1984. - Effects of Permethrin on the behavior of individually tagged honey bees, Apis mellifera L. (Hymenoptera : apidae). Environ. Entomol., 13, 375-378.

GeOrge D.A., RincKer C.M., 1985. - Results and conclusions of using pesticides with the alfalfa leaf cutting bee in the production of alfalfa seed. J. Agric. Entomol., 2, 93-97.

Shricker B., StePhen W.P., 1970. - The effect of sublethal doses of parathion on honeybee behaviour. I. Oral administration and the communication dance. J. apic. Res., 9, 141-153.

Smirle M.J., Winston M.L., Woodward K.L., 1984. - Development of a sensitive bioassay for evaluating sublethal pesticide effects on the honey bee (Hymenoptera Apidae). J. econ. Entomol., 77, 63-67.

TASei J.N., Carre S., 1985. - Effets du traitement de luzerne en fleurs (Medicago sativa L.) avec de la deltaméthrine et de la phosalone sur l'abeille solitaire: Megachile rotundata L. (Hym. Megachilidae). Acta Ecologica Ecol. Applic., 6, 165-173.

Tasei J.N., Carre S., Bosio P.G., Debray Ph., Hariot J., 1987(a). - Effects of alphacypermethrin (WL $85871=$ Fastac) and phosalone (Zolone) on adults and progeny of the leaf-cutting bee : Megachile rotundata F. (Hymenoptera Megachilidae) pollinator of lucerne (Medicago sativa L.). Pestic. Sci., 21, 119-128.

Tasei J.N., Carre S., Grondeau C., Hureau J.M., 1987(b). - Effets d'applications insecticides à l'égard d'apoïdes pollinisateurs autres que l'abeille domestique : (Megachile rotundata F. et Bombus terrestris L.), Conf. intern. sur les ravageurs en agriculture, Paris, 1, 2, 3 déc. 1987, A.N.P.P., 127136.

TоRсніо P.F., 1983. - The effects of field application of naled and trichlorfon on the alfalfa leafcutting bee, Megachile rotundata F. J. Kansas Entomol. Soc., 56, 62-68.

WALLER G.D., 1969. - Susceptibility of an alfalfa leafcutting bee to residues of insecticides on foliage. $J$. econ. Entomol., 62, 189-192. 